\title{
A supplementary note on constructing the general Earth's rotation theory
}

\author{
Victor A. Brumberg and Tamara V. Ivanova \\ Institute of Applied Astronomy, \\ St.-Petersburg, 191187, Russia \\ email: vabrumberg@verizon.net, itv@ipa.nw.ru
}

\begin{abstract}
Representing a post-scriptum supplementary to a previous paper of the authors Brumberg \& Ivanova (2011) this note aims to simplify the practical development of the Earth's rotation theory, in the framework of the general planetary theory, avoiding the non-physical secular terms and involving the separation of the fast and slow angular variables, both for planetary-lunar motion and Earth's rotation. In this combined treatment of motion and rotation, the fast angular terms are related to the mean orbital longitudes of the bodies, the diurnal and Euler rotations of the Earth. The slow angular terms are due to the motions of pericenters and nodes, as well as the precession of the Earth. The combined system of the equations of motion for the principal planets and the Moon and the equations of the Earth's rotation is reduced to the autonomous secular system with theoretically possible solution in a trigonometric form. In the above-mentioned paper, the Earth's rotation has been treated in Euler parameters. The trivial change of the Euler parameters to their small declinations from some nominal values may improve the practical efficiency of the normalization of the Earth's rotation equations. This technique may be applied to any three-axial rigid planet. The initial terms of the corresponding expansions are given in the Appendix.
\end{abstract}

Keywords. celestial mechanics, methods: analytical, Earth: rotation

This note represents a post-scriptum remark to our paper Brumberg \& Ivanova (2011) (later denoted by BI) to improve the practical efficiency of the normalization of the Earth's rotation equations. The seven first-order differential equations of the Earth's rotation treated in this paper in the formula (2.45) typical for General Planetary Theory (GPT) are:

$$
\dot{X}=i \mathcal{N}[P X+R(X, t)]
$$

where $X$ and $R$ stand for 7 -vectors of the variables and right-hand members, respectively, $X=\left(X_{i}\right)=\left(u, \bar{u}, v, \bar{v}, m^{\prime}, \bar{m}^{\prime}, m_{3}\right)$, and $R=\left(R_{i}\right), \quad(i=1,2, \ldots, 7)$.

The first four components of $X$ represent the Euler parameters related to the ordinary Euler angles $\varphi, \psi, \theta$ by means of the relations : $u=-\sin \frac{\theta}{2} e^{-i \frac{\psi+\varphi}{2}}, v=i \cos \frac{\theta}{2} e^{i \frac{\psi-\varphi}{2}}$ and interrelated by the identity:

$$
u \bar{u}+v \bar{v} \equiv 1 .
$$

The last three components of $X$ are small dimensionless quantities determining the components of the angular Earth's rotation velocity $\omega=\left(\omega_{i}\right),(i=1,2,3)$ by means of $\omega_{1}=\Omega m_{1}, \omega_{2}=\Omega m_{2}, \omega_{3}=\Omega\left(1+m_{3}\right), \Omega$ being the mean Earth's rotation velocity. In consistency with our previous papers $\Omega=-2 n, n=$ const.

With $I_{1}, I_{2}, I_{3}$ denoting the Earth's principal inertia moments, $k_{1}=\frac{I_{3}-I_{1}}{2 I_{2}}$ and $k_{2}=\frac{I_{3}-I_{2}}{2 I_{1}}$, we write:

$$
m_{1}=2 \sqrt{k_{2}} m_{1}^{\prime}, \quad m_{2}=2 \sqrt{k_{1}} m_{2}^{\prime} \quad m=m_{1}+i m_{2} \quad m^{\prime}=m_{1}^{\prime}+i m_{2}^{\prime} .
$$


The right-hand members are:

$$
\begin{array}{ll}
R_{1}=m_{3} u-m \bar{v} & R_{2}=-\bar{R}_{1}, \\
R_{3}=m_{3} v+m \bar{u}, & R_{4}=-\bar{R}_{3} \\
R_{5}=-4 \sqrt{k_{1} k_{2}} m_{3} m^{\prime}-\frac{1}{4 n^{2}}\left(\frac{1}{\sqrt{k_{1}}} \frac{\mathcal{M}_{2}}{I_{2}}-\frac{i}{\sqrt{k_{2}}} \frac{\mathcal{M}_{1}}{I_{1}}\right) & R_{6}=-\bar{R}_{5} \\
R_{7}=2 \sqrt{k_{1} k_{2}} \frac{I_{1}-I_{2}}{I_{3}}\left(m^{\prime 2}-\bar{m}^{\prime 2}\right)+\frac{i}{2 n^{2}} \frac{\mathcal{M}_{3}}{I_{3}}, &
\end{array}
$$

$\mathcal{M}_{1}, \mathcal{M}_{2}, \mathcal{M}_{3}$ being the components of the torque vector. Their expressions and expansion techniques are given in $\mathrm{BI}$.

At last, $\mathcal{N}$ and $P$ are $7 \times 7$ diagonal matrices with the structure $\mathcal{N}=\operatorname{diag}(n, n, n, n, n$, $n, n), P=\operatorname{diag}(1,-1,1,-1, \epsilon,-\epsilon, 0)$ with $\epsilon=-4 \sqrt{k_{1} k_{2}}$. The main frequencies of $u, v$ and $m^{\prime}$ are $n, n$ and $n \epsilon$, respectively (the last frequency corresponds to the Euler period of the Earth's rotation). The frequency for $m_{3}$ is zero.

In BI, the Earth's rotation system (1) was treated together with the system describing the planetary-lunar motion in the framework of GPT. Due to the identity (2) and the specific features of the right-hand members, there were no difficulties related to the peculiarities of $P$. However, from the practical point of view, it might be more adequate not to deal with variables $u, v$ themselves but rather to use their small declinations from some nominal values. The simplest option seems to choose, as a nominal initial approximation, $\underset{0}{X}$ satisfying the equations: $\underset{0}{X}=i \mathcal{N} P \underset{0}{X}$. The components of $\underset{0}{X}$ are:

$$
\underset{0}{u}=a e^{i n t}, \underset{0}{v}=b e^{i n t}, \underset{0}{m^{\prime}}=c e^{i \epsilon n t}, \underset{0}{m_{3}}=0,
$$

with constant $a, b, c$ (the zero value for $m_{3}$ can be always provided by the choice of $\Omega$ ).

In terms of the Euler angles, this nominal approximation corresponds to $\varphi=-2 n t+\varphi_{0}$, $\psi=\psi_{0}, \theta=\theta_{0}, \varphi_{0}, \psi_{0}, \theta_{0}$ being constants.

Moreover, similarly to (2), we obtain: $a \bar{a}+b \bar{b} \equiv 1$ with

$$
a=-\sin \frac{\theta_{0}}{2} e^{-i \frac{\psi_{0}+\varphi_{0}}{2}}, b=i \cos \frac{\theta_{0}}{2} e^{i \frac{\psi_{0}-\varphi_{0}}{2}}, c=-\frac{1}{4 n}\left(\frac{\left(\omega_{1}\right)_{0}}{\sqrt{k_{2}}}+i \frac{\left(\omega_{2}\right)_{0}}{\sqrt{k_{1}}}\right),
$$

$\left(\omega_{j}\right)_{0}(j=1,2)$ being the components of the angular Earth's rotation velocity for the initial moment of time.

Returning now to the original equations (1), let us define $X=\underset{0}{X}+\delta X$ reducing (1) to:

$$
\delta \dot{X}=i \mathcal{N}[P \delta X+R \underset{0}{X}+\delta X, t)] .
$$

Equations (1) and (5) have the same form but, in contrast to $X$, the components $\delta X$ are small quantities. The right-hand members in (1) are trigonometric functions of the mean longitudes of the planets and the Moon. In addition, the right-hand members in (5) have two trigonometric arguments $n t$ and $\epsilon n t$ related to the Earth's rotation. With numerical values for $\varphi_{0}, \psi_{0}, \theta_{0}$ and $c$, one can easily get the right-hand member expansions generalizing those treated in BI. Afterwards, one may solve (5) in traditional manner, by using Picard-type iterations, in powers of $\delta X$ or in more sophisticated manner, by combining (5) with the planetary-lunar GPT equations and finding the normalizing transformation to the combined secular system, as it was done in BI. It may be noted that the right-hand member $R$ in (5) is holomorphic with respect to $\delta X$ with the zero- and first-degree terms proportional to the small perturbing parameters. Such terms involve the corresponding 
contributions into the normalization transformation described by (4.8)-(4.12) of BI but do not interfere with the resulting secular system.

\section{References}

Brumberg, V. \& Ivanova, T. 2011, Cel. Mech. Dyn. Astron. 109, 385

\section{Appendix}

In Appendix we give a set of formulas to compute the initial terms of the expansions for the Earth's rotation variables (or any other three-axial rigid planet). Using (3) for the variable $m$ and the nominal solution (4), we obtain:

$$
\begin{aligned}
\delta \dot{u} & =i n\left(\delta u-m_{0} \bar{v}\right)=i n \delta u-i n \bar{b}\left[\left(\sqrt{k_{2}}+\sqrt{k_{1}}\right) c e^{i(-1+\epsilon) n t}+\left(\sqrt{k_{2}}-\sqrt{k_{1}}\right) \bar{c} e^{i(-1-\epsilon) n t}\right], \\
\delta \dot{v} & =i n(\delta v+\underset{0}{m} \bar{u})=i n \delta v+i n \bar{a}\left[\left(\sqrt{k_{2}}+\sqrt{k_{1}}\right) c e^{i(-1+\epsilon) n t}+\left(\sqrt{k_{2}}-\sqrt{k_{1}}\right) \bar{c} e^{i(-1-\epsilon) n t}\right], \\
\delta \dot{m}^{\prime} & =i n \epsilon \delta m^{\prime}-\frac{1}{4 n}\left(\frac{1}{\sqrt{k_{2}}} \frac{\mathcal{M}_{1}}{I_{1}}+\frac{i}{\sqrt{k_{1}}} \frac{\mathcal{M}_{2}}{I_{2}}\right), \\
\delta \dot{m}_{3} & =-\frac{1}{2} i n \epsilon \frac{I_{1}-I_{2}}{I_{3}}\left(c^{2} e^{2 i \epsilon n t}-\bar{c}^{2} e^{-2 i \epsilon n t}\right)-\frac{1}{2 n} \frac{\mathcal{M}_{3}}{I_{3}}
\end{aligned}
$$

resulting to

$$
\begin{aligned}
\delta u & =\bar{b}\left[c \frac{\sqrt{k_{2}}+\sqrt{k_{1}}}{2-\epsilon} e^{i(-1+\epsilon) n t}+\bar{c} \frac{\sqrt{k_{2}}-\sqrt{k_{1}}}{2+\epsilon} e^{i(-1-\epsilon) n t}\right], \\
\delta v & =-\bar{a}\left[c \frac{\sqrt{k_{2}}+\sqrt{k_{1}}}{2-\epsilon} e^{i(-1+\epsilon) n t}+\bar{c} \frac{\sqrt{k_{2}}-\sqrt{k_{1}}}{2+\epsilon} e^{i(-1-\epsilon) n t}\right], \\
\delta m^{\prime} & =-\frac{1}{4 n}\left[\int\left(\frac{1}{\sqrt{k_{2}}} \frac{\mathcal{M}_{1}}{I_{1}}+\frac{i}{\sqrt{k_{1}}} \frac{\mathcal{M}_{2}}{I_{2}}\right) e^{-i \epsilon n t} d t\right] e^{i \epsilon n t}, \\
\delta m_{3} & =-\frac{1}{4} \frac{I_{1}-I_{2}}{I_{3}}\left(c^{2} e^{2 i \epsilon n t}+\bar{c}^{2} e^{-2 i \epsilon n t}\right)-\frac{1}{2 n} \int \frac{\mathcal{M}_{3}}{I_{3}} d t .
\end{aligned}
$$

In addition, we demonstrate the initial terms of $\delta m^{\prime}$ and $\delta m_{3}$ expansions provided that the Sun and the Moon move in planar circular orbits.

$$
\begin{aligned}
\delta m^{\prime}= & \left(1+\epsilon_{1}\right)(1-2 a \bar{a})\left[a b \frac{K C_{1}+L C_{2}}{2-\epsilon} e^{2 i n t}+\bar{a} \bar{b} \frac{K C_{2}+L C_{1}}{2+\epsilon} e^{-2 i n t}\right] \\
& +\sum_{j=3,9}\left(\delta_{j 3}+\epsilon_{1} \delta_{j 9}\right) \times\left[a \bar{b}^{3} \frac{K C_{2}+L C_{1}}{2+2 n_{j}^{\prime}+\epsilon} e^{-2 i\left(n t+\lambda_{j}\right)}-\bar{a}^{3} b \frac{K C_{2}+L C_{1}}{2-2 n_{j}^{\prime}+\epsilon} e^{-2 i\left(n t-\lambda_{j}\right)}\right. \\
& \left.-a^{3} \bar{b} \frac{K C_{1}+L C_{2}}{2-2 n_{j}^{\prime}-\epsilon} e^{2 i\left(n t-\lambda_{j}\right)}+\bar{a} b^{3} \frac{K C_{1}+L C_{2}}{2+2 n_{j}^{\prime}-\epsilon} e^{2 i\left(n t+\lambda_{j}\right)}\right] \\
& +\frac{1}{2} \sigma C_{1}\left\{a \bar{b}^{3} L\left[\frac{e^{-i\left(2 n t+\lambda_{3}+\lambda_{9}\right)}}{2+n_{3}^{\prime}+n_{9}^{\prime}+\epsilon}+5 \frac{e^{-i\left(2 n t+3 \lambda_{3}-\lambda_{9}\right)}}{2+3 n_{3}^{\prime}-n_{9}^{\prime}+\epsilon}\right]\right. \\
& +\bar{a} b^{3} K\left[\frac{e^{i\left(2 n t+\lambda_{3}+\lambda_{9}\right)}}{2+n_{3}^{\prime}+n_{9}^{\prime}-\epsilon}+5 \frac{e^{i\left(2 n t+3 \lambda_{3}-\lambda_{9}\right)}}{2+3 n_{3}^{\prime}-n_{9}^{\prime}-\epsilon}\right] \\
& -\bar{a}^{3} b L\left[\frac{e^{-i\left(2 n t-\lambda_{3}-\lambda_{9}\right)}}{2-n_{3}^{\prime}-n_{9}^{\prime}+\epsilon}+5 \frac{e^{-i\left(2 n t-3 \lambda_{3}+\lambda_{9}\right)}}{2-3 n_{3}^{\prime}+n_{9}^{\prime}+\epsilon}\right]
\end{aligned}
$$




$$
\begin{aligned}
& -a^{3} \bar{b} K\left[\frac{e^{i\left(2 n t-\lambda_{3}-\lambda_{9}\right)}}{2-n_{3}^{\prime}-n_{9}^{\prime}-\epsilon}+5 \frac{e^{i\left(2 n t-3 \lambda_{3}+\lambda_{9}\right)}}{2-3 n_{3}^{\prime}+n_{9}^{\prime}-\epsilon}\right] \\
& +3(1-2 a \bar{a}) \bar{a} \bar{b} L\left[\frac{e^{-i\left(2 n t+\lambda_{3}-\lambda_{9}\right)}}{2+n_{3}^{\prime}-n_{9}^{\prime}+\epsilon}+\frac{e^{-i\left(2 n t-\lambda_{3}+\lambda_{9}\right)}}{2-n_{3}^{\prime}+n_{9}^{\prime}+\epsilon}\right] \\
& \left.+3(1-2 a \bar{a}) a b K\left[\frac{e^{i\left(2 n t+\lambda_{3}-\lambda_{9}\right)}}{2+n_{3}^{\prime}-n_{9}^{\prime}-\epsilon}+\frac{e^{i\left(2 n t-\lambda_{3}+\lambda_{9}\right)}}{2-n_{3}^{\prime}+n_{9}^{\prime}-\epsilon}\right]\right\}, \\
& \delta m_{3}=B+\bar{B}, \\
& B=L\left\{-\frac{1}{2}\left(1+\epsilon_{1}\right) a^{2} b^{2} e^{4 i n t}+\sum_{j=3,9} \frac{1}{2}\left(\delta_{j 3}+\epsilon_{1} \delta_{j 9}\right)\left[a^{4} \frac{e^{2 i\left(2 n t-\lambda_{j}\right)}}{2-n_{j}^{\prime}}+b^{4} \frac{e^{2 i\left(2 n t+\lambda_{j}\right)}}{2+n_{j}^{\prime}}\right]\right. \\
& +\frac{1}{2} \sigma\left\{a^{4}\left[\frac{e^{i\left(4 n t-\lambda_{3}-\lambda_{9}\right)}}{4-n_{3}^{\prime}-n_{9}^{\prime}}+5 \frac{e^{i\left(4 n t-3 \lambda_{3}+\lambda_{9}\right)}}{4-3 n_{3}^{\prime}+n_{9}^{\prime}}\right]+b^{4}\left[\frac{e^{i\left(4 n t+\lambda_{3}+\lambda_{9}\right)}}{4+n_{3}^{\prime}+n_{9}^{\prime}}+5 \frac{e^{i\left(4 n t+3 \lambda_{3}-\lambda_{9}\right)}}{4+3 n_{3}^{\prime}-n_{9}^{\prime}}\right]\right. \\
& \left.\left.-6 a^{2} b^{2}\left[\frac{e^{i\left(4 n t+\lambda_{3}-\lambda_{9}\right)}}{4+n_{3}^{\prime}-n_{9}^{\prime}}+\frac{e^{i\left(4 n t-\lambda_{3}+\lambda_{9}\right)}}{4-n_{3}^{\prime}+n_{9}^{\prime}}\right]\right\}\right\}-\frac{1}{4} \frac{I_{1}-I_{2}}{I_{3}} c^{2} e^{2 i \epsilon n t} \text {. }
\end{aligned}
$$

In these expressions,

$$
\begin{aligned}
C_{1} & =\frac{I_{3}\left(I_{1} \sqrt{k_{2}}+I_{2} \sqrt{k_{1}}\right)}{2 I_{1} I_{2} \sqrt{k_{1} k_{2}}}, & C_{2} & =\frac{I_{3}\left(I_{2} \sqrt{k_{1}}-I_{1} \sqrt{k_{2}}\right)}{2 I_{1} I_{2} \sqrt{k_{1} k_{2}}}, \\
K & =-\frac{3}{4}\left(1-\frac{I_{1}+I_{2}}{2 I_{3}}\right) \frac{G M_{0}}{A_{3}^{3} n^{2}}, & L & =-\frac{3}{8} \frac{I_{1}-I_{2}}{I_{3}} \frac{G M_{0}}{A_{3}^{3} n^{2}}, \\
\varepsilon_{1} & =\frac{M_{9}}{M_{0}}\left(\frac{A_{3}}{A_{9}}\right)^{3}, & \sigma & =\frac{M_{9}}{M_{3}} \frac{A_{9}}{A_{3}} .
\end{aligned}
$$

$n_{j}^{\prime}=\frac{n_{j}}{n}(j=3,9), M_{0}$ is the mass of the Sun, $M_{j}, A_{j}, \lambda_{j}$ denote masses, semi-major axes and mean longitudes of the Earth-Moon barycentre (for $j=3$ ) and the Moon (for $j=9$ ), respectively, $\delta_{i j}$ being the Kronecker symbol.

The resulting expansions (6) and (7) are obtained in the literal form with respect to two small parameters $K$ and $L$. Needless to say, they have the trigonometric form with respect to time. 